\title{
Intermédialités
}

Histoire et théorie des arts, des lettres et des techniques

Intermediality

History and Theory of the Arts, Literature and Technologies

\section{L’image est le mouvant}

\section{Georges Didi-Huberman}

Numéro 3, printemps 2004

Devenir-Bergson

Becoming-Bergson

URI : https://id.erudit.org/iderudit/1005466ar

DOI : https://doi.org/10.7202/1005466ar

Aller au sommaire du numéro

Éditeur(s)

Centre de recherche sur l'intermédialité

ISSN

1705-8546 (imprimé)

1920-3136 (numérique)

Découvrir la revue

Citer cet article

Didi-Huberman, G. (2004). L’image est le mouvant. Intermédialités /

Intermediality, (3), 11-30. https://doi.org/10.7202/1005466ar
Résumé de l'article

À l'époque où triomphait, dans les laboratoires de biologie, la méthode expérimentale théorisée par Claude Bernard, Henri Bergson a proposé une " philosophie expérimentale » dont le réel comme apparaître constituait l'objet central. Contrepoint à une véritable épistémologie des limites du scientisme, la proposition bergsonienne place les images au centre du connaître. À travers une mise en parallèle des modèles bergsoniens et des images instrumentalisées par Étienne-Jules Marey, ce texte questionne la constitution de l'image-temps à la fin du XIX ${ }^{\mathrm{e}}$ siècle. 


\title{
L'image est le mouvant
}

\author{
GEORGES DIDI-HUBERMAN
}

\begin{abstract}
$\dot{A}$
l'époque même où triomphait, dans les laboratoires de biologie, la méthode expérimentale théorisée par Claude Bernard, Henri Bergson a proposé d'«aller chercher l'expérience à sa source, ou plutôt au-dessus de ce tournant décisif où, s'infléchissant [...], elle devient proprement l'expérience humaine ${ }^{1}$. N Non que Bergson refusât la leçon de Claude Bernard. Il a loué, au contraire, sa modestie devant le réel, sa façon de respecter la singularité des phénomènes, c'est-à-dire leur multiplicité et leur complexité. Il en admirait, corrélativement, l'extraordinaire audace philosophique : celle de refuser, au nom de l'expérience, tout esprit de système (et Bergson de citer ce dictum de Claude Bernard: «La philosophie et la science ne doivent pas être systématiques») pour produire d'authentiques concepts expérimentaux qui seront des concepts fluides ou, du moins, souples, plastiques: «Rappelons-nous aussi que jamais une idée, si souple que nous l'ayons faite, n'aura la même souplesse que les choses. Soyons donc prêts à l'abandonner pour une autre, qui serrera l'expérience de plus près encore 2 ."

La philosophie, dit ailleurs Bergson,

n'est proprement elle-même que lorsqu'elle dépasse le concept, ou du moins lorsqu'elle s'affranchit des concepts raides et tout faits pour créer des concepts bien différents de ceux que nous manions d'habitude, je veux dire des représentations souples, mobiles, presque fluides, toujours prêtes à se mouler sur les formes fuyantes
\end{abstract}

1. Henri Bergson, Matière et mémoire. Essai sur la relation du corps à l'esprit [1896], dans (Euvres, André Robinet (éd.), Paris, Presses universitaires de France, 1970 [1959], p. 321. Désormais, les références à cet ouvrage seront indiquées par le sigle «MM» suivi de la page et placées entre parenthèses dans le corps du texte.

2. Henri Bergson, «La philosophie de Claude Bernard» [1913], La pensée et le mouvant [1934], dans Euvres, p. 1438-1439. 
d'un réel que l'analyse seule reste incapable d'appréhender en ses mouvements singuliers³. Mais qu'est-ce qu'une pensée du réel capable de renoncer, non seulement aux systèmes en général, mais encore à la raideur - si ce n’est à la rigueur - des concepts eux-mêmes?

La réponse à cette question, chaque lecteur de Bergson s'en souvient comme d'une expérience de pensée proprement sidérante lorsque, au début de Matière et mémoire, le jeune philosophe demande que nous nous placions dans la situation - typiquement expérimentale - de «feindre pour un instant que nous ne connaissions rien ", de renoncer à tous nos ancrages dans les systèmes philosophiques existants - réalistes ou idéalistes, peu importe - et, par conséquent, de nous «en tenir » d'abord «aux apparences ». Que se passe-t-il alors? «Me voici donc en présence d'images, au sens le plus vague que l'on puisse prendre ce mot, images perçues quand j'ouvre mes sens, inaperçues quand je les ferme. » (Mм, p. 169-170)

On imaginerait volontiers, à l'énoncé de telles phrases, tous les grands fondateurs de systèmes, depuis Platon jusqu'à Auguste Comte, se retournant, furieux, dans leur tombe. Qu'est-ce donc qu'une philosophie qui demande que soit maintenue l'apparence afin de mieux penser l'apparaître et, dans son flux, la structure même du réel? Qu'est-ce qu'une philosophie qui ose s'en remettre aux images jusque dans leur «sens le plus vague » et qui ne craint pas, quelques pages plus loin, d'affirmer: "Ce sont des objets ou, si l'on aime mieux, des images »... ( $\mathrm{Mm}, \mathrm{p} .173)$ Et même: "J'appelle matière l'ensemble des images. » (MM, p. 215) Qu'est-ce qu'une philosophie qui fait s'interpénétrer la perception et la mémoire jusqu'à revendiquer la notion de « représentation inconsciente »? (MM, p. $213-215$, p. $283-291$ )

L'audace de cette entrée en matière philosophique, dans le premier chapitre de Matière et mémoire, n’a rien des emportements métaphysiques ou "vitalistes» où l'on enferme un peu trop facilement le style de la pensée bergsonienne. Cette audace, en effet, donne la contrepartie rigoureuse d'une véritable épistémologie en formation, c'est-à-dire d'une réflexion sur les enjeux et les limites de la science positive. Bien avant Bachelard, Bergson a posé le problème de la démarche scientifique en termes d'obstacles ou de «faux problèmes »; bien avant Althusser, il a souligné les méfaits de la «philosophie

3. Henri Bergson, «Introduction à la métaphysique » [1903], La pensée et le mouvant, p. 1401-1402. Désormais, les références à ce texte seront indiquées par le sigle «IM » suivi de la page et placées entre parenthèses dans le corps du texte. 
spontanée des savants » en opposant la pauvreté du scientisme à la richesse de la science elle-même ${ }^{4}$. Aussi le philosophe devra-t-il repenser ce qu'expérience veut dire à l'âge triomphant de la méthode expérimentale, ce qui suppose un rapport nouveau de la pensée au savoir:

À première vue, il peut paraître prudent d'abandonner à la science positive la considération des faits. La physique et la chimie s'occuperont de la matière brute, les sciences biologiques et psychologiques étudieront les manifestations de la vie. La tâche du philosophe est alors nettement circonscrite. Il reçoit, des mains du savant, les faits et les lois, et, soit qu'il cherche à les dépasser pour en atteindre les causes profondes, soit qu'il croie impossible d'aller plus loin et qu'il le prouve par l'analyse même de la connaissance scientifique, dans les deux cas il a pour les faits et pour les relations, tels que la science les lui transmet, le respect que l'on doit à la chose jugée. À cette connaissance il superposera une critique de la faculté de connaître et aussi, le cas échéant, une métaphysique: quant à la connaissance même, dans sa matérialité, il la tient pour affaire de science et non pas de philosophie.

Mais comment ne pas voir que cette prétendue division du travail revient à tout brouiller et à tout confondre? La métaphysique ou la critique que le philosophe se réserve de faire, il va les recevoir toutes faites de la science positive, déjà contenues dans les descriptions et les analyses dont il a abandonné au savant tout le souci. Pour n'avoir pas voulu intervenir, dès le début, dans les questions de fait, il se trouve réduit, dans les questions de principe, à formuler purement et simplement en termes plus précis la métaphysique et la critique inconscientes, partant inconsistantes, que dessine l'attitude même de la science vis-à-vis de la réalité5.

Et Bergson de nous prévenir (comme pour une première justification de ses audaces théoriques) : «La philosophie envahit ainsi le domaine de l'expérience. [...] Il en résultera d'abord une certaine confusion. » (EC, p. 663) Qu'est-ce à dire? Que la philosophie, en ce domaine, ne gagnera sa précision - c'est tout l'enjeu revendiqué dans les premières pages de La pensée et le mouvant ${ }^{6}$ - qu'à

4. Henri Bergson, «Introduction (deuxième partie) : de la position des problèmes » [1922], La pensée et le mouvant, p. 1271-1330.

5. Henri Bergson, L'évolution créatrice [1907], dans (Euvres, p. 66o. Désormais, les références à cet ouvrage seront indiquées par le sigle «EC» suivi de la page et placées entre parenthèses dans le corps du texte.

6. Henri Bergson, «Introduction (première partie) : croissance de la vérité, mouvement rétrograde du vrai » [1922], La pensée et le mouvant, p. 1253-1256. 
faire exploser les cadres habituels de son rapport à la science en créant l'inévitable confusion d'un déplacement des limites entre les méthodes et les domaines établis de la connaissance. L’idée que la philosophie soit quelque chose comme une «synthèse de la science » exprimée dans la langue naturelle s'avère, aux yeux de Bergson, aussi «désobligeante pour la science» (n’ayant pas besoin qu'on tire pour elle les conclusions de sa propre démarche) qu'elle est «injurieuse pour la philosophie» (n'ayant pas besoin qu'on dise pour elle ce qui est réel et ce qui ne l'est pas) ${ }^{7}$.

D’où vient, exactement, cette nécessaire confusion que le travail philosophique devrait être capable de transformer en instrument de précision? Elle vient de ce que Bergson n’hésite pas à affronter le réel - la matière, la vie, le mouvement, la durée, la conscience, la mémoire - à travers l'image de son apparaître, l'image en tant qu'elle ne se réduit pas à une simple perception. Telle est la décision philosophique majeure ${ }^{8}$. Décision qui entraîne un bouleversement méthodologique du savoir lui-même : une chose stable (déjà apparue et non disparue, comme fixée dans l'être) est susceptible d'analyse, puisqu'on peut tourner autour d'elle et produire une suite réglée de points de vue à son sujet; tandis que l'image de l'apparaître (l'infixable apparition, la chose apparaissante) n'est susceptible que d'une intuition, mouvement de connaissance par lequel on est contraint d' «entrer» dans l'image sous peine de manquer complètement la chose. (IM, p. 1392-1396)

Connaître par images, c'est donc approcher l'apparaître des choses en deçà du fait observable. C'est toucher la singularité en deçà de toute loi généralisa-

7. Henri Bergson, «L'intuition philosophique »[1911], La pensée et le mouvant, p. 13581360.

8. Reconnue par quelques exégètes de la pensée bergsonienne. Voir à ce sujet Lydie Adolphe, La dialectique des images chez Bergson, Paris, Presses universitaires de France, 1951; Paul Naulin, "Le problème de la conscience et la notion d'“image" », dans Bergson: naissance d'une philosophie. Actes du colloque de Clermont-Ferrand, Paris, Presses universitaires de France, 1990, p. 97-109; Jean-François Bordron, «Bergson et les images. L'iconicité de la pensée dans "Le possible et le réel” ", dans Lire Bergson: "Le possible et le réel », Frédéric Cossutta (dir.), Paris, Presses universitaires de France, 1998, p. 159181) et, surtout, prolongée dans toute la philosophie deleuzienne de l'image. Voir notamment Gilles Deleuze, Cinéma 1. L'image-mouvement, Paris, Éditions de Minuit, 1983; Gilles Deleuze, Cinéma 2. L'image-temps, Paris, Éditions de Minuit, 1985). Mais, quoi qu'en dise Deleuze, la tentative bergsonienne s'apparente en bien des points à l'approche - sinon à la réduction - phénoménologique de l'apparaître. 
trice. «Un brin d'herbe ne ressemble pas plus à un autre brin d'herbe qu'un Raphaël à un Rembrandt ${ }^{9}$. "Écrivant cela, Bergson ne songe évidemment pas à mettre en doute les classifications botaniques en usage; mais il demande pour chaque brin d'être, si j'ose dire, que soit reconnu le style particulier, dans une temporalité et dans un contexte à chaque fois différents, de son apparition. Voilà pourquoi la connaissance par images se situe en deçà de la représentation et de l'appauvrissement que celle-ci impose - par abstraction, par schématisme, par "pré-vision» - à l'imprévisible mouvement de l'apparaître ${ }^{10}$. Un botaniste peut fort bien énoncer, sur le plan classificatoire, l'identité des deux brins d'herbe; le philosophe bergsonien préférera, plus proche en cela de l'historien d'art, réfléchir sur leurs singularités, leurs ressemblances et leurs dissemblances ${ }^{11}$.

Connaître par images, c'est renoncer à la synthèse du «tout fait» et se risquer l'intuition - fatalement provisoire, mais rythmée sur le temps en acte $\mathrm{du}$ «se faisant» (EC, p. 696). C'est atteindre ce qui, dans chaque système, «vaut mieux que le système et lui survit» (EC, p. 697). C’est découvrir qu'au delà des arrangements ou des «juxtapositions » dont le réel serait mécaniquement et intemporellement composé, nous devons rendre compte d'une perpétuelle création, c'est-à-dire d'un imprévisible réarrangement de toutes choses, qui procède par interpénétration de toutes ces choses entre elles, dans l'espace comme dans le temps (EC, p. 698-699, p. 782-783).

On aboutit dès lors au paradoxe fécond d'une pratique de la pensée définie comme «faculté de voir immanente » au mouvement et à la durée, une pratique où «l'entendement lui-même, en se soumettant à une certaine discipline, pourrait préparer une philosophie qui le dépasse» (EC, p. 494, p. 707), c'est-àdire une philosophie capable de se soustraire à la pétrification du système, voire à la rigidité du concept. Lorsque Bergson en appelle à l'«adhésion » du concept à son objet - contre les «conceptions si abstraites, et par conséquent si vastes qu'on y ferait tenir tout le possible, et même l'impossible ${ }^{12} »-$, il signifie clairement, sans peur des paradoxes, qu'un concept digne de ce nom doit être un

9. Henri Bergson, «Le possible et le réel » [1930], La pensée et le mouvant, p. 1343.

10. Henri Bergson, «Le possible et le réel», p. 1331.

11. «[...] l'identité est du géométrique et la ressemblance du vital. La première relève de la mesure, l'autre est plutôt du domaine de l'art. » (Henri Bergson, «Introduction (deuxième partie) », La pensée et le mouvant, p. 1299)

12. Henri Bergson, "Introduction (première partie)", La pensée et le mouvant, p. 1253 . 
instrument singulier, "moulable» sur son seul objet et, par conséquent, rétif à toute généralisation bien que capable de la fluidité et de la plasticité dont le plâtre ou la pâte à modeler font preuve sur la forme toujours différente à laquelle ils s'appliquent ${ }^{13}$.

Connaître par images serait donc toucher le réel par le biais même, le pouvoir d'immanence dont les images sont le véhicule privilégié: moulage de la chose (comme le plâtre de Rodin, à l'époque de Bergson, retenait la singularité du moindre élément de son vocabulaire formel) et modulation du milieu (comme la palette de Monet, à la même époque, reconfigurait la fluidité et les nuances du moindre plan d'eau des Nymphéas).

Mais ce que l'image gagne en précision et en nuances, en singularité et en multiplicités, en «moulage» et en «modulations», elle le perd en universalité (puisque le moulage ne s'adapte qu'à l'unique forme dont il offre le négatif) et en stabilité (puisqu'une modulation ne cesse jamais de varier). Ce serait là comme un principe d'incertitude avant la lettre: le même phénomène ne peut pas être simultanément observé sous ses deux régimes fondamentaux, corpusculaire (mécanique) et ondulatoire (dynamique). L'image, parce qu'elle se moule sur la singularité des phénomènes, peut en restituer les «mille nuances» (IM, p. 1398); mais son adhérence même, sa capacité d'immanence, l'empêchent de nous donner une idée continue, stable ou éternisée, du réel (et c'est bien pourquoi, selon Bergson, il faut entièrement repenser les rapports entre science et métaphysique). (IM, p. 1419-1432) L'image et l'intuition, qui échouent là où se montrent efficaces le concept et la synthèse, réussissent là où achoppent le concept et la synthèse: elles «élargissent notre perception » en respectant les différences, les nuances, les mouvements et les moindres changements qualitatifs de la réalitée ${ }^{14}$. Mais il y a deux contreparties au moins: la chose disparaît

13. Henri Bergson, «L'intuition philosophique», p. 1361. Il faut rappeler que Bergson oppose, sur ce point, la fluidité des concepts analytiques et expérimentaux d'Aristote à la rigidité des concepts systématiques et idéaux de Platon. Voir Henri Bergson, «La vie et l'œuvre de Ravaisson" [1904], La pensée et le mouvant, p. 1452-1456.

14. Henri Bergson, «La perception du changement» [1911], La pensée et le mouvant, p. $1365-1392$. 


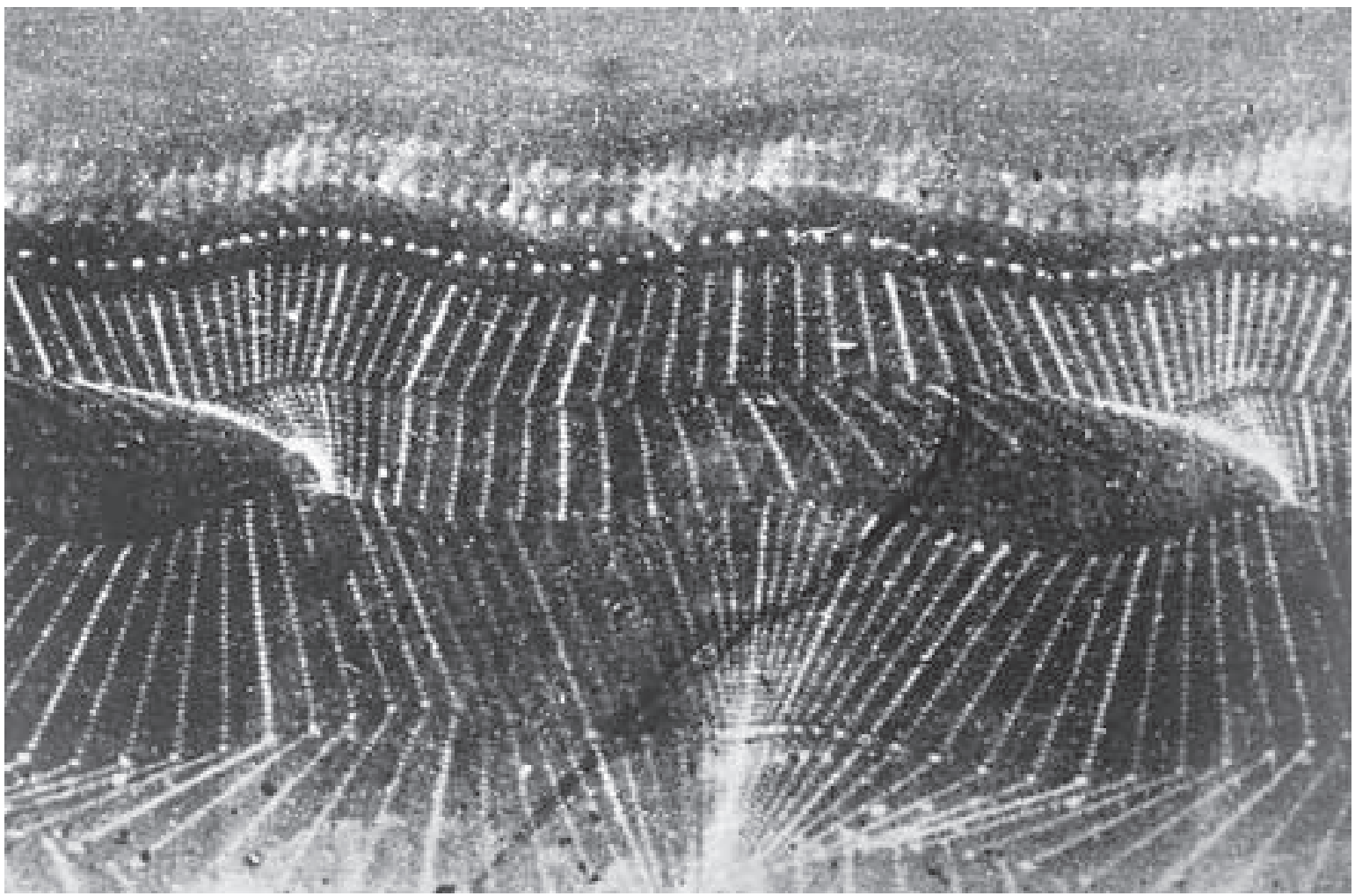

Fig. 1. Étienne-Jules Marey, Marche de l'homme (portant un habit noir à lignes blanches), 1884. Chronophotographie sur plaque fixe.

dans sa stabilité, dans sa quantité ou sa mesure, dans sa nature de «coupe immobile», au profit d'une durée continue qui forme, en quelque sorte, le milieu mouvant de l'apparaître; le temps de connaître devient cependant luimême un temps discontinu, instable, intuitif, saccadé, évanouissant puisque «moulé» sur le temps singulier de l'apparaître:

[...] penser intuitivement est penser en durée. L'intelligence part ordinairement de l'immobile, et reconstruit tant bien que mal le mouvement avec des immobilités juxtaposées. L'intuition part du mouvement, le pose ou plutôt l'aperçoit comme la réalité même, et ne voit dans l'immobilité qu'un moment abstrait, instantané pris par notre esprit sur une mobilité. L'intelligence se donne ordinairement des choses, entendant par là du stable, et fait du changement un accident qui s'y surajouterait. Pour l'intuition l'essentiel est le changement: quant à la chose, telle que l'intelligence l'entend, c'est une coupe pratiquée au milieu du devenir et érigée par notre 
esprit en substitut de l'ensemble. [...] L'intuition, attachée à une durée qui est croissance, y perçoit une continuité ininterrompue d'imprévisible nouveauté ${ }^{15}$.

L'intuition est là cependant, mais vague et surtout discontinue. C'est une lampe presque éteinte, qui ne se ranime que de loin en loin, pour quelques instants à peine. [...] De ces intuitions évanouissantes, et qui n'éclairent leur objet que de distance en distance, le philosophe doit s'emparer, d'abord pour les soutenir, ensuite pour les dilater et les raccorder ainsi entre elles. (EC, p. 722)

Nous n'avons sous les yeux, à bien les regarder, que des choses mouvantes: le monde est le mouvant. Mais comment connaître les mouvements mêmes du mouvant? Il semble que Bergson, ici, nous mette face à une contradiction: d'un côté, il faut renoncer à penser le mouvement en termes discontinus, cesser de réduire le mouvement à des «instantanés » ou à des «immobilités juxtaposées»; d'un autre côté, la saisie du mouvement - l'intuition, l'image - ne se fait que sur le mode du «vague» et «surtout [du] discontinu». Notre pensée, écrit bien Bergson, n'éclaire le phénomène que comme «une lampe presque éteinte, qui ne se ranime que de loin en loin, pour quelques instants à peine. » L'intuition capte le mouvant pour autant que, comme lui - puisqu'elle lui est immanente - , elle passe, telle un papillon, apparaissant et s' «évanouissant » presque aussitôt dans le ciel opaque de l'intelligence humaine. L'image «élargit la perception» des choses; mais le prix à payer tient dans la singularité, donc dans la fragilité et la passagèreté même - pour parler comme Freud - de l'image $^{16}$. Ce n'est pas un hasard si Bergson demande que «nous nous enfoncions [dans la perception des phénomènes] pour la creuser et l'élargir», à l'instar des tableaux de Turner lorsqu'ils appréhendent justement, en inlassables variantes, les «dissolving views» du vent, de la brume ou de la tempête ${ }^{17}$.

15. Henri Bergson, «Introduction (deuxième partie)», La pensée et le mouvant, p. 1275.

16. C'est bien pourquoi Bergson ne revendique l'image qu'à la multiplier : telles, les images de l'«écoulement», puis du «déroulement d'un rouleau» ou d'une pelote de fil, puis d'un «spectre aux mille nuances», puis d'un «élastique infiniment petit», etc. (IM, p. $1396-1403$ )

17. Henri Bergson, «La perception du changement» [1911], La pensée et le mouvant, p. $1368-1372$. 


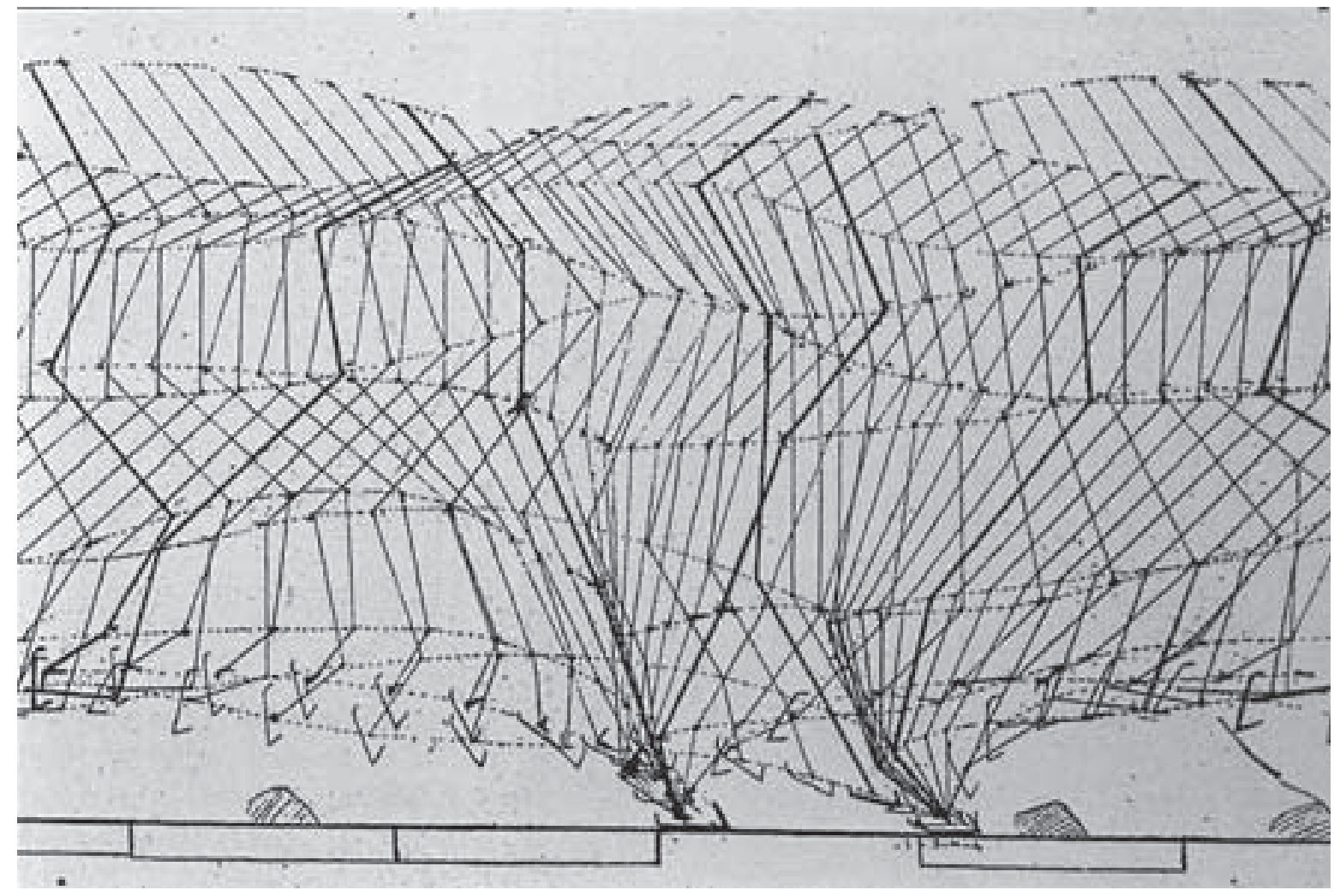

Fig. 2. Étienne-Jules Marey, Cheval au pas : épure géométrique, 1885. Dessin à l'encre.

Le paradigme bergsonien de la «lampe presque éteinte» serait donc - comme l'anticipait bien, dans l'Essai sur les données immédiates de la conscience, la question du phénomène envisagé sous l'angle de son intensité - un paradigme esthésique et même esthétique ${ }^{18}$. On ne s'étonnera pas, soit dit en passant, de voir resurgir ce paradigme à des moments cruciaux du discours artistique lorsqu'il s'agit, précisément, d'affirmer que quelque chose de nouveau est en train d'apparaître. Ainsi Jean Paulhan évoquera-t-il une «petite aventure en pleine nuit» - allumer puis éteindre aussitôt une lampe, pour tâtonner dans l'espace

18. Henri Bergson, Essai sur les données immédiates de la conscience [1889], dans (Euvres, p. 5-17, où la question de l'intensité se trouve renvoyée, d'emblée, à l'exemple des «sentiments profonds» et du «sentiment esthétique». 
entrevu d'un appartement - aux fins d'exposer la valeur bouleversante de ce que fait voir, selon lui, l'espace cubiste ${ }^{19}$. Ainsi Tony Smith, plus tard, référerat-il sa nécessité de sculpter des volumes minimalistes à l'expérience sensorielle d'un déplacement nocturne tout à coup privé de son éclairage habituel, sur une autoroute en construction du New Jersey ${ }^{20}$.

Husserl, bien plus tôt, avait tenté de se forger un concept de la «représentation vide» en questionnant, dans le même exemple de «la lumière qu'on éteint», ce que l'on voit encore (comme image) dans ce qui vient juste de disparaître (comme phénomène)21. Mais il est une autre réalité - tout aussi philosophique - à laquelle la configuration bergsonienne nous ramène plus directement encore : c'est un dispositif instrumental fait d'images qui apparaissent pour disparaître aussitôt; il vise la continuité du mouvement mais, pour la rendre visible, procède à l'artifice d'une intermittence stroboscopique. Ce dispositif, né à l'époque de Bergson, offrait l'expérience singulière d'un régime visuel dominé par le caractère saccadé des images passantes et de l'intensité lumineuse elle-même, comme si une lampe s'éteignait et se rallumait obstinément sur le monde visible à chaque tour de manivelle de l'opérateur. Cette expérience est celle de la cinématographie.

Dans son chapitre introductif de L'image-mouvement, Gilles Deleuze a, pour ainsi dire, «sauvé» les critiques bergsoniennes de l’ «illusion cinématographique» en montrant qu'elles permettaient, dans le cadre plus général d'une philosophie du mouvement et du temps, de rendre le cinéma un peu mieux pensable $^{22}$. De fait, Bergson semble avoir réfuté le cinéma comme quelqu'un qui tiendrait entre ses mains un bout de pellicule où le mouvement, en effet, se trouve réduit aux «coupes immobiles » des photogrammes, plutôt qu'un film projeté où surgissent bien ce que Deleuze nomme, par différence, des « coupes mobiles». Et Deleuze de renchérir : «La reproduction de l'illusion n'est-elle pas aussi sa correction, d'une certaine manière? Peut-on conclure de l'artificialité des moyens à l'artificialité du résultat ${ }^{23}$ ? » Bref, le film comme support a beau

19. Jean Paulhan, La peinture cubiste, Paris, Éditions Gallimard, 1990 [1942-1956], p. $61-81$.

20. Tony Smith, cité et commenté dans Georges Didi-Huberman, Ce que nous voyons, ce qui nous regarde, Paris, Éditions de Minuit, 1992, p. 61-84.

21. Edmund Husserl, La représentation vide, trad. Jocelyn Benoist, Paris, Presses universitaire de France, 2003 [1913], p. 11-36.

22. Gilles Deleuze, Cinéma 1. L’image-mouvement, p. 9-22.

23. Gilles Deleuze, Cinéma 1. L’image-mouvement, p. 10. 


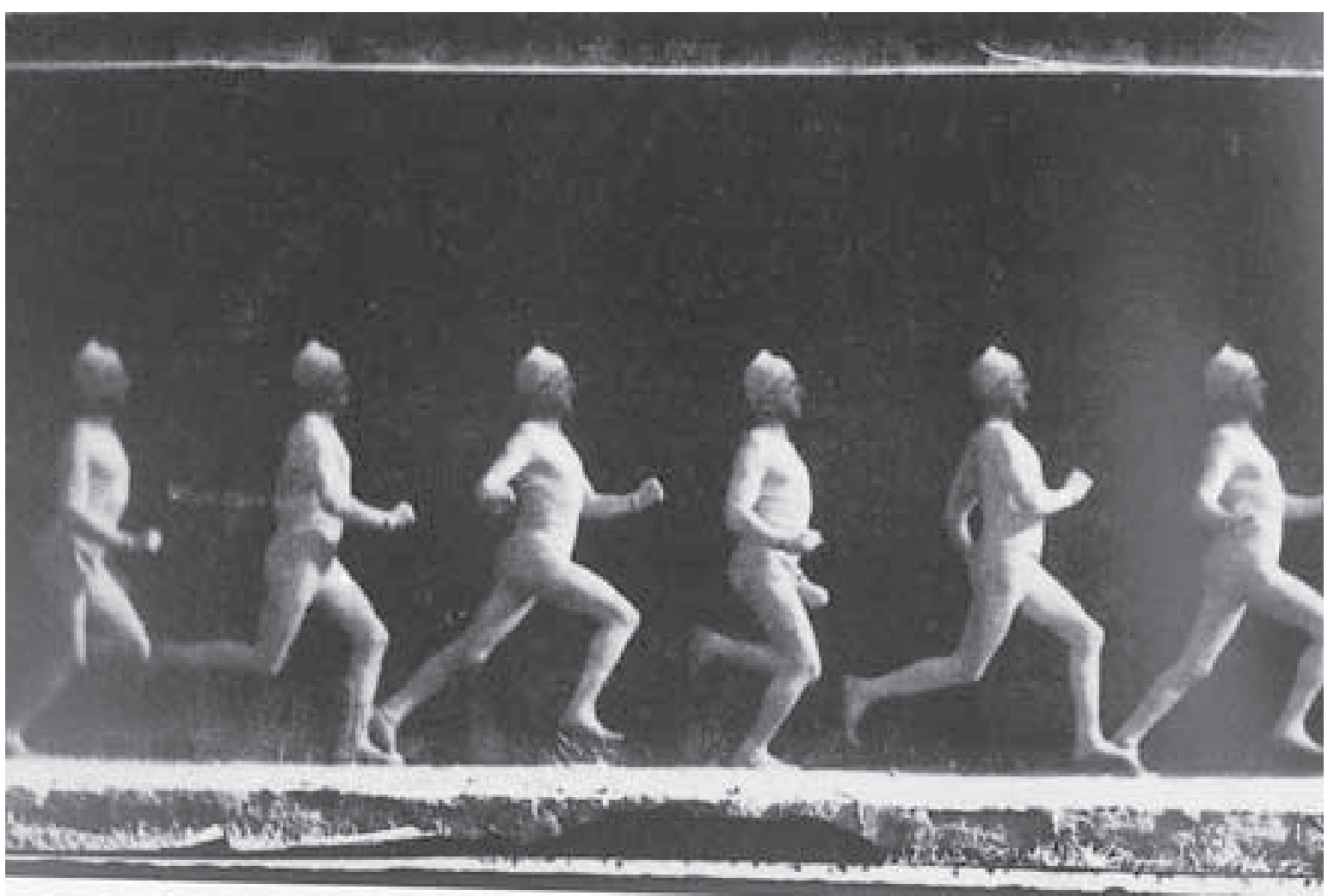

Fig. 3. Étienne-Jules Marey, Course de l'homme (portant un habit blanc), 1883. Chronophotographie sur plaque fixe.

constituer, en termes bergsoniens, un mensonge sur le mouvement, le film déroulé en projection peut offrir l'instrument par excellence pour retrouver la vérité du mouvement comme «donnée immédiate ${ }^{24} »$. Deleuze ira jusqu’à prétendre que seul le cinéma «moderne» - à partir de Rossellini - aura été capable d'incarner cette image-temps qu'appelle de ses vœux toute la pensée de Bergson ${ }^{25}$.

Il faut cependant revenir, me semble-t-il, aux conditions mêmes dans lesquelles Bergson dut confronter sa propre notion d'image mouvante ou d'imagedurée à ce qu'il appelait une illusion cinématographique. Que visait-il donc, exactement, dans le mot «cinématographie»? En 1907, le lecteur de L'évolution créatrice - où se trouve le fameux chapitre final sur «Le mécanisme

24. Gilles Deleuze, Cinéma 1. L’image-mouvement, p. 11. 
cinématographique de la pensée et l'illusion mécanistique » (EC, p. 725-807) pouvait voir une production déjà considérable de courts-métrages réalisés par les frères Lumière: un millier de films étaient déjà dans leur catalogue de 1905; et pas moins de cinquante-quatre films de Georges Méliès - dont Pochardiana ou le rêveur éveillé, La toile d'araignée merveilleuse, Hallucinations pharmaceutiques ou le truc du potard ou encore La poupée vivante - furent produits, pour la seule année 1907, au théâtre Robert-Houdin ${ }^{26}$.

Mais Bergson a aussi voulu préciser que son analogie «cinématographique» remontait au moins à un cours donné au Collège de France en 19021903: "Nous y comparions le mécanisme de la pensée conceptuelle à celui du cinématographe» (EC, p. 725 n.), note-t-il. L'intelligence comme instrumentalisation artificielle de la durée, la pensée mécanistique comme réduction du mouvement aux simples découpages d'instantanés ou d'arrangements spatiaux, tous ces thèmes de L'évolution créatrice (EC, p. 508, p. 613) ne sont pas nouveaux chez Bergson, loin de là: ils se mettent en place à une époque où les frères Lumière étaient encore loin d'avoir déposé leur brevet de «Cinématographe». Non seulement on les trouve en 1896 dans Matière et mémoire (MM, p. 324-329; p. 337-343) mais, déjà, en 1889, dans l'Essai sur les données immédiates de la conscience, lorsque Bergson analysait les deux conceptions antagonistes de la durée en critiquant la réduction mécaniste des phénomènes intensifs à de simples problèmes d'extension, de mesure donc de divisibilité27.

Inversement, on demeure surpris qu'aucune allusion au cinéma comique ne soit faite dans Le rire, en 1900, alors que les nombreuses Vues comiques des Lumière et les farces cinématographiques de Méliès - aux titres toujours succulents: Spiritisme abracadabrant (1900), Le déshabillage impossible (1900) ou encore Le savant et le chimpanzé (1900), pour ne prendre que des exemples tournés cette année-là - révélaient déjà toute leur efficacité de «mécanique plaqué[e] sur du vivant ${ }^{28}$ ». Lorsque, plus tard, Bergson continuera de critiquer

25. Gilles Deleuze, Cinéma 2. L'image-temps, p. 62-164 et p. 354-359.

26. Voir Bernard Chardère, Les images Lumière, Paris, Éditions Gallimard, 1995, p. 191-198. Jacques Malthête et Laurent Mannoni (dirs.), Méliès. Magie et cinéma, Paris, Éditions Paris-Musées, 2002, p. 261-264.

27. Voir Henri Bergson, Essai sur les données immédiates de la conscience, p. 3, p. 8, p. 72-80, p. 93-109, etc.

28. Henri Bergson, Le rire. Essai sur la signification du comique [1900], dans CEuvres, p. 405 et passim. 


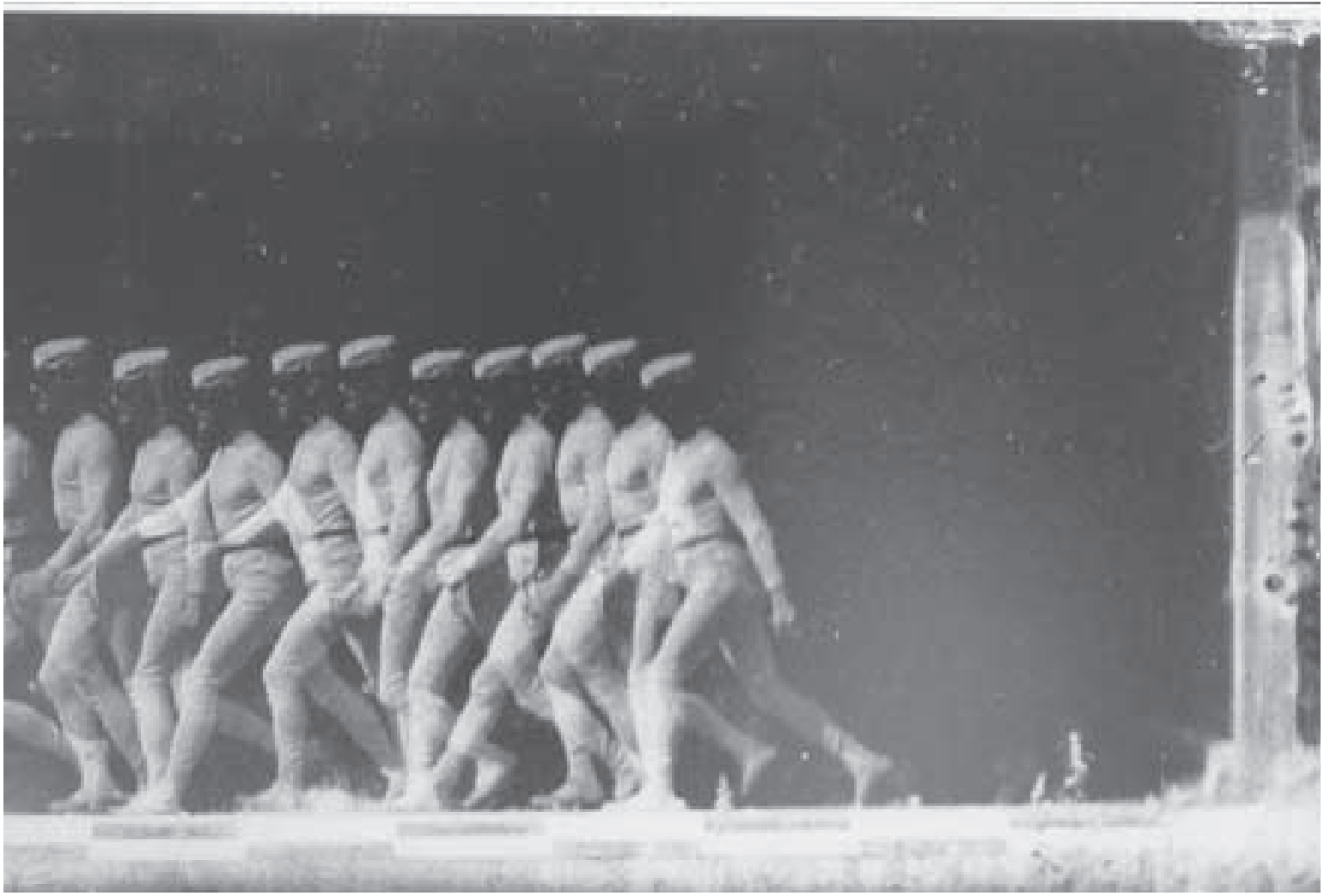

Fig. 4. Étienne-Jules Marey, Marche de l'homme (portant un habit blanc), 1883. Chronophotographie sur plaque fixe.

les «instantanés pris par notre entendement sur la continuité du mouvement et de la durée", lorsqu'il fustigera cette «succession semblable à celle des images d'un film cinématographique» que l'intelligence reste «condamnée à détailler $[\ldots]$ image par image ${ }^{29} »-$ nous devrons comprendre, une fois pour toutes, que ce n'est en rien vers le «septième art» que s'est orientée la critique bergsonienne - pourtant esthétique en son fond - de la cinématographie.

Pour Bergson, en effet, l'image cinématographique fut à l'image mouvante ce que toute connaissance mécaniste (abstraite, extérieure) était à la connaissance dynamique (concrète, inhérente) des phénomènes. La «cinématographie»,

29. Henri Bergson, «Introduction (première partie)», La pensée et le mouvant, p. 1258-126o. 
telle que l'entend Bergson, relève d'un paradigme gnoséologique et non du monde goguenard que présentaient, à la fin du XIX siècle, les théâtres forains des grands boulevards investis par l'invention d'Edison et de Lumière. Parce qu'elle est toujours évoquée dans le contexte d'une philosophie de la connaissance et de la sensorialité, la «cinématographie » bergsonienne doit donc s'entendre selon l'acception stricte que lui donna Lucien Bull, en 1928, dans son livre justement intitulé La cinématographie: c'est un instrument expérimental de connaissance visuelle basé sur la décomposition analytique du mouvement et visant sa synthèse théorique ${ }^{30}$.

Lucien Bull fut le dernier assistant d'Étienne-Jules Marey et, plus encore, son «fils spirituel ${ }^{31}$ ». C'est donc bien du côté de la chronophotographie et de Marey lui-même qu'il faut situer ce que Bergson a voulu entendre par cinématographie. Lorsque le philosophe entra au Collège de France, le grand physiologiste y enseignait depuis une trentaine d'années déjà. Ils n'étaient sans doute pas faits pour se comprendre jusqu'au bout, mais ils ne pouvaient pas s'ignorer. On a quelquefois l'impression que La pensée et le mouvant fut écrit pour répondre au livre de Marey sur Le mouvement, que L'évolution créatrice inverse toutes les propositions de La machine animale, et que Matière et mémoire cherche à réfuter jusqu'au bout La méthode graphique - cette mnémotechnique des phénomènes - revendiquée par le savant mécanicien ${ }^{32}$. Bergson et Marey auront, néanmoins, siégé dans les mêmes sociétés savantes et cosigné un programme d'études expérimentales sur certains phénomènes «psychophysiques» liés à l'hypnose et à la vogue spirite du XIX ${ }^{\mathrm{e}}$ siècle $^{33}$.

30. Lucien Bull, La cinématographie, Paris, Éditions Armand Colin, 1928, p. VIIVIII et $1-33$.

31. Voir Laurent Mannoni, Étienne-Jules Marey. La mémoire de l'œil, Paris, Milan, Cinémathèque française, Mazzotta, 1999, p. 383-390.

32. Voir Étienne-Jules Marey, La machine animale, Paris, Baillière, 1873 ; ÉtienneJules Marey, La méthode graphique dans les sciences expérimentales et particulièrement en physiologie et en médecine, Paris, Masson, 1878 et Étienne-Jules Marey, Le mouvement, Paris, Masson, 1894.

33. Henri Bergon, Étienne-Jules Marey et al., «Groupe d’études de phénomènes psychiques» [1901], Mélanges, André Robinet (éd.), Paris, Presses universitaires de France, 1972 , p. 509-510. 


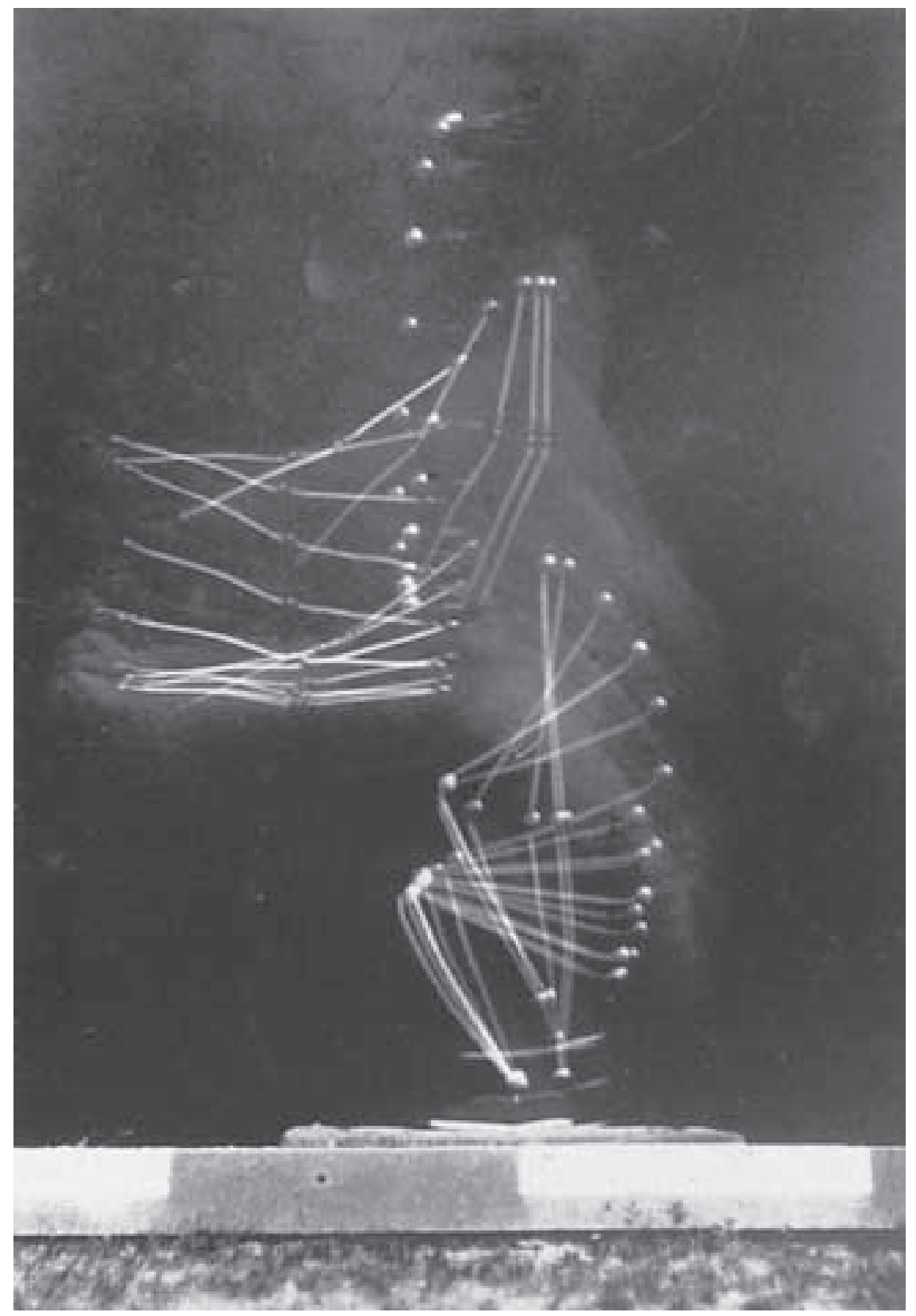

Fig. 5. Étienne-Jules Marey, Flexion des genoux, bras tendus (homme portant un habit noir à lignes et points blancs), 1884. Chronophotographie sur plaque fixe. 
Marey n'est jamais cité dans les livres de Bergson, mais les allusions à son œuvre y fleurissent indubitablement. Lorsque, dans l'Essai sur les données immédiates de la conscience, Bergson affirme que le mouvement n'est pas plus divisible que la durée n'est mesurable, c'est toute la tentative de Marey - avec sa fragmentation visuelle des gestes et sa volonté concomitante de mesure qui se trouve philosophiquement mise en cause; lorsque Bergson critique avec force «ceux qui se plaisent à juxtaposer les états [et] à en former une chaîne ou une ligne », il semble rejeter les séries chronophotographiques de Marey comme les innombrables courbes destinées à offrir une trace lisible - indiciaire et géométrique tout à la fois - des phénomènes vitaux ${ }^{34}$. (fig. 1 et 2)

Certaines comparaisons théoriques, dans Matière et mémoire, donnent à penser que Bergson les imagina pour avoir eu sous les yeux les planches produites par Marey à la même époque. Ainsi, les «mille positions successives d'un coureur» forment l'objet commun du questionnement, chez le philosophe et le physiologiste; mais Bergson y revendique le «changement en profondeur» (ou ce qu'il avait déjà nommé des «multiplicités internes»), contre le changement «localisé çà et là, mais en surface » qu'il diagnostique sans doute dans les protocoles expérimentaux de Marey. (MM, p. 343-344) (fig. 3 et 4) Dans d'autres pages du même livre, Bergson réfute tout ce qui sert à Marey pour capter, mesurer et synthétiser la dynamique des élans vitaux: «[...] les axes ou les points auxquels on le rapporte» n'expriment pas plus le mouvement d'un corps que les éléments juxtaposés d'une série chronophotographique (fig. 5); la ligne «divisible [et] dépourvue de qualité » ne décrit rien de plus que ne le font des «symboles mathématiques», puisqu'il s'agit, dans les deux cas, de «parquer le mouvement dans l'espace » en ignorant sa réelle temporalité, sa rythmicité propre (fig. 6); Bergson va même jusqu'à évoquer l' «écran noir sur lequel se détacherait l'image», exactement comme on le voit dans les procédures photographiques mises au point par Marey dans sa «station » expérimentale. (MM, p. 188, p. 329-332 et p. 351-352. Je souligne)

Il était donc cohérent que, dans L'évolution créatrice, l'«illusion cinématographique » fût exprimée dans les termes - typiquement mareysiens — de «vues instantanées » qui prétendent saisir le mouvement en juxtaposant dans l'espace une série de «coupes immobiles»:

34. Henri Bergson, Essai sur les données immédiates de la conscience, p. 69-80, que l'on peut comparer, notamment, avec Étienne-Jules Marey, La méthode graphique, p. $1-106$. 


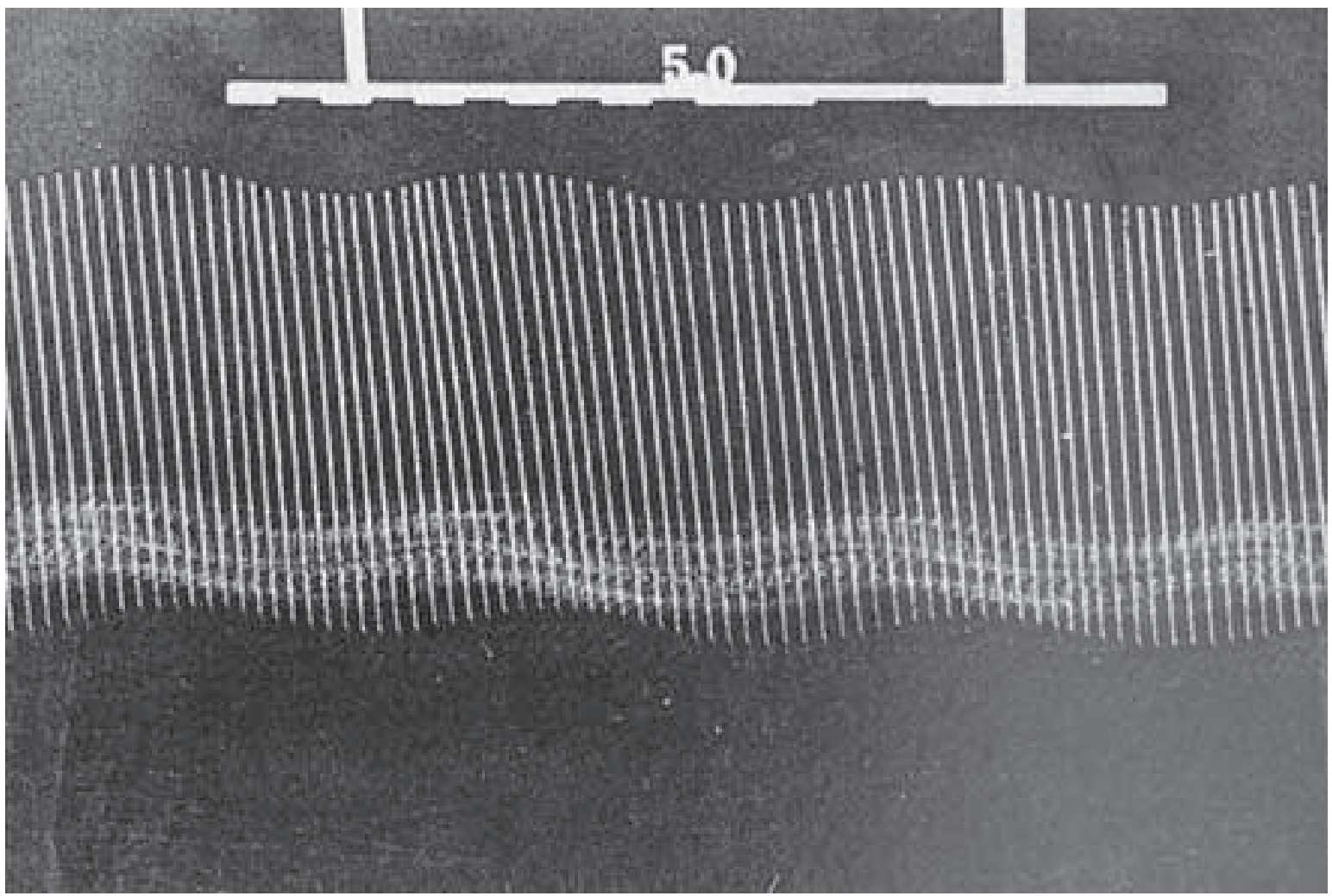

Fig. 6. Étienne-Jules Marey, Marche d'un homme (portant un habit noir et une baguette blanche fixée le long de la colonne vertébrale), 1886. Chronophotographie sur plaque fixe.

[...] l'intelligence, comme les sens, se borne à prendre, de loin en loin, sur le devenir de la matière, des vues instantanées et, par là même, immobiles. [...] Ainsi se détachent de la durée les moments qui nous intéressent et que nous avons cueillis le long de son parcours. Nous ne retenons qu'eux [et] nous devenons incapables de voir l'évolution vraie, le devenir radical. Nous n'apercevons du devenir que des états, de la durée que des instants et, même quand nous parlons de durée et de devenir, c'est à autre chose que nous pensons. Telle est la plus frappante des illusions que nous voulons examiner. Elle consiste à croire qu'on pourra penser l'instable par l'intermédiaire du stable, le mouvant par l'immobile. (EC, p. 726)

Or, la vie est une évolution. Nous concentrons une période de cette évolution en une vue stable que nous appelons une forme, et, quand le changement est devenu assez considérable pour vaincre l'heureuse inertie de notre 
perception, nous disons que le corps a changé de forme. Mais, en réalité, le corps change de forme à tout instant. Ou plutôt il n'a pas de forme, puisque la forme est de l'immobile et que la réalité est mouvement. Ce qui est réel, c'est le changement continuel de forme: la forme n'est qu'un instantané pris sur une transition. Donc, ici encore, notre perception s'arrange pour solidifier en images discontinues la continuité fluide du réel. (EC, p. 750)

Que cette illusion soit efficace comme un tour de prestidigitation - façon Méliès ou Robert Houdin - , c'est ce que montrera l' «artifice du cinématographe» décrit une fois par Bergson dans sa dimension spectaculaire de film projeté sur un écran: artifice, en effet, puisqu'il ne restituerait du mouvement singulier qu'une épure de "mouvement en général » intégrée à l'appareil mécanique. (EC, p. 752-753) Non seulement cette analyse fait l'impasse sur l'expérience cinématographique concrète - puisqu'elle en nie l'indicialité, c'est-à-dire la capacité à restituer des mouvements singuliers - , mais encore elle amènera Bergson à proférer, plus tard, l'argument absurde selon lequel « le film pourrait se dérouler dix fois, cent fois, mille fois plus vite sans que rien fût modifié à ce qu'il déroule ${ }^{35}$.»

Cette relative inattention au cinéma comme expérience sensorielle spécifique montre, s'il en était besoin, que le problème, pour Bergson, était ailleurs. Nous avons pu lire comment, dans L'évolution créatrice, c'est la question séculaire de la forme et du temps qui fait le nœud, le cœur de son travail critique. «La forme n'est qu'un instantané pris sur une transition»: dans cette phrase, le mot «instantané » dénote une invention photographique récente - la mise au point des plaques « extra-rapides » au gélatino-bromure d'argent — d'où Marey aura précisément tiré toute l'efficacité de sa méthode instrumentale ${ }^{36}$; mais le mot «forme » renvoie, lui, à une très longue tradition philosophique qui remonte au moins à Platon. Or, c'est bien à la forme comme eidos ou idea, comme abstraction métaphysique, que Bergson s'en prenait d'abord (presque un siècle avant Derrida).

35. Henri Bergson, «Introduction (première partie)", La pensée et le mouvant, p. 1259-1260. Cet argument est, en fait, calqué sur celui que Bergson donne ailleurs à propos de la connaissance mécaniste : les lois de la physique ne changeraient en rien si le monde allait dix fois plus vite ou dix fois plus lentement qu'il ne va en réalité, preuve que ces lois manquent bien l'élément concret de la durée.

36. Voir Michel Frizot, Étienne-Jules Marey: chronophotographie, Paris, NathanDelpire, 2001, p. 103-107. 


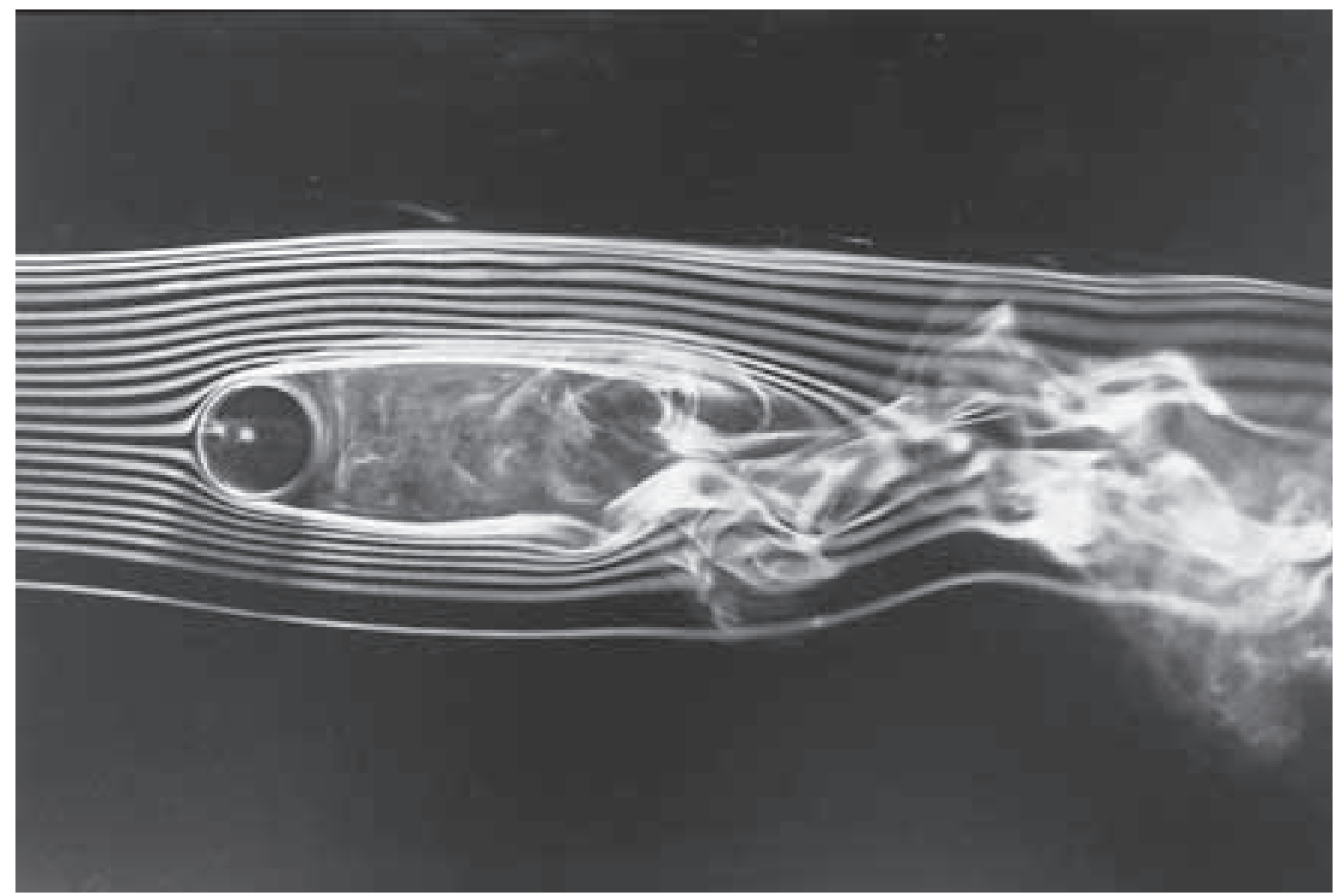

Fig. 7. Étienne-Jules Marey, Études des mouvements de l'air par la méthode des filets de fumée, 1900-1901. Photographie instantanée.

Voilà pourquoi il n'était pas choquant, à ses yeux, d'utiliser d'autres comparaisons bien moins «modernes» que celle du cinématographe: le kaléidoscope, où chaque configuration (forme) ne doit pas faire oublier la secousse (temps) qui l'a engendrée; la mosaïque, où la réunion de tous les éléments (travail divisé de la forme) ne réussira jamais à restituer l'élan simple du dessin de maître (œuvre indivisible du temps) qui lui a servi de modèle; jusqu'aux basreliefs du Parthénon, où Bergson voit «le même mécanisme cinématographique » à l'œuvre, si ce n'est que la métaphysique antique isolait des «attitudes caractéristiques» là où la science moderne travaille avec des «instants quelconques » qu'elle «met tous au même rang» dans la même loi physique ou sur la même planche chronophotographique. (EC, p. 571-572, p. 754, p. 775-776) 
On comprend alors en quoi la «cinématographie» désigne moins un instrument spécifique qu'une instrumentalisation très générale où l'apparaître se trouve, en quelque sorte, capturé et nié. La chronophotographie de Marey n'est que l'exemple contemporain d'une mythologie de la connaissance vivace depuis que les philosophes éléates ont voulu rabattre tout mouvement sur l'espace parcouru par le mobile ${ }^{37}$. Marey offre, pour Bergson, l'incarnation d'un Argus moderne qui voudrait que jamais la lampe ne s'éteigne et que jamais l'œil ne se ferme: l'«illusion cinématographique» consistant à croire tout voir, et à manipuler ce tout vu comme une étendue divisible à loisir, une quantité intégralement géométrisable, une énergie vitale aisément instrumentalisable.

Or, nous savons par expérience que l'apparaître, y compris dans sa dimension visuelle, est durée vitale: c'est-à-dire, non qu'il dure comme chose durcie, raide, prétendument stable et susceptible, à ce titre, de donner lieu à quelque idée éternelle; mais qu'il dure comme ondoie simplement le flux dansant, inlassable, de la vague. Plus profondément, l'apparaître dure, au sens de Bergson, en ce qu'il passe en se survivant dans une image-mouvance. (MM, p. 276-316) Encore faut-il savoir - et tel serait l'objet même de la "philosophie expérimentale » proposée par Bergson - tirer une connaissance inhérente à ce passage, à cette survivance, à cette mouvance ou sillage de l'apparaître ${ }^{38}$ (fig. 7)...

37. Henri Bergson, Essai sur les données immédiates de la conscience, p. 75-76.

38. Je développe cette notion de «sillage » dans un article qui prend appui sur celuici et le prolonge : Georges Didi-Huberman, «L'image-sillage », L'inactuel, $\mathrm{n}^{\circ} 10$ [à parâtre]. 